\title{
DOPPLER MICROEMBOLIC SIGNALS IN PATIENTS WITH TWO DIFFERENT TYPES OF BILEAFLET VALVES
}

Dimitrios Georgiadis, MD $^{\mathrm{a}}$

Saskia Braun ${ }^{\mathrm{a}}$

Frank Uhlmann ${ }^{\mathrm{a}}$

Gillian M. Bernacca, $\mathrm{PhD}^{\mathrm{c}}$

Wilhelm Johannes Schulte-Mattler, MD

Stephan Zierz, MD

Hans Reinhard Zerkowski, MD
Objectives: This study was performed to evaluate the prevalence and counts of Doppler microembolic signals in patients with St Jude Medical valves (St Jude Medical, Inc, St Paul, Minn) and patients with ATS valves (ATS Medical, Inc, Minneapolis, Minn) and their relation to clinical parameters.

Methods: A total of 179 outpatients of the department of cardiothoracic surgery were examined. They included 98 men and 81 women, aged $61 \pm 11$ years, with ATS $(n=91)$ or St Jude Medical $(n=88)$ valves in the aortic $(n=110)$, mitral $(n=39)$, or both positions $(n=30)$. Neurologic examination was followed by transcranial Doppler monitoring for microembolic signals. Monitoring was performed bilaterally over the middle cerebral arteries for 1 hour per session.

Results: Microembolic signal counts and prevalence were significantly higher in patients with St Jude Medical as compared with ATS valves. Valve type and presence of diabetes mellitus were the only predictors of microembolic signal prevalence on multivariate analysis. No influence of microembolic signals on cerebral embolic complications was established. Additionally, patients with a postoperative history of cerebral embolic complications did not have a higher number of microembolic signals than remaining patients. Interobserver variability was satisfactory.

Conclusions: Patients with St Jude Medical valves were shown to have significantly higher microembolic signal counts than patients with ATS valves. However, our results suggest that microembolic signal counts cannot be used to predict cerebral embolic complications. Their relation to neuropsychologic deficits remains to be evaluated. (J Thorac Cardiovasc Surg 2001;121:1101-6)
$\mathrm{T}^{\mathrm{s}}$ he potential of transcranial Doppler sonography in the detection of microembolic signals (MES) has been described in several stroke-prone patient groups, including patients with prosthetic cardiac valves. The prognostic relevance of MES concerning prediction of clinically manifest brain embolism was shown to be marginal ${ }^{1,2}$ or even lacking. ${ }^{3-5}$ Still, a relation between MES counts and prevalence of neuropsychologic deficit in persons with artificial heart valves was recently described. ${ }^{6}$ This issue is of particular interest, because significant differences in

From the Department of Neurology a and the Department of Cardiothoracic Surgery, ${ }^{\mathrm{b}}$ Martin-Luther-University HalleWittenberg, Halle/Saale, Germany, and the Department of Cardiac Surgery, Royal Infirmary, University of Glasgow, ${ }^{\mathrm{c}}$ Glasgow, United Kingdom.

Copyright (c) 2001 by The American Association for Thoracic Surgery

$0022-5223 / 2001 \$ 35.00+0 \quad \mathbf{1 2 / 1 / 1 1 3 1 7 6}$

doi: $10.1067 / \mathrm{mtc} .2001 .113176$
MES counts depending on the type of prosthetic valve inserted have been reported. ${ }^{1-4}$

The purpose of this study was the comparison of MES prevalence and counts between patients having St Jude Medical valves (SJM; St Jude Medical, Inc, St Paul, Minn) and patients having the ATS valve (ATS Medical, Inc, Minneapolis, Minn).

\section{Patients and methods}

Patients. The medical records of all patients operated on between January 1995 and December 1997 were reviewed. Four cardiac surgeons, using a standardized technique, had performed all operations. Valves were inserted in the supraannular position. ATS standard, ATS standard "soft-cuff," ATS-AP, SJM standard, SJM-HP, or SJM Masters prostheses were implanted in all patients. No anatomic or clinical criteria were applied for the choice of valve. Valves were chosen at the discretion of the cardiac surgeon, on the basis of personal preference.

All patients had undergone examination of the extracranial arteries supplying the brain with continuous wave Doppler 
and - in selected cases - duplex ultrasound as part of the routine preoperative assessment. Presence of hemodynamically significant carotid disease ( $>70 \%$ luminal stenosis on continuous wave Doppler ultrasound), as well as perioperative stroke, served as exclusion criteria. A total of 239 patients were identified and received a written invitation to participate in this study. Of these invitees, 191 patients attended, 12 $(6.3 \%)$ of whom were excluded because of insufficient temporal bone window. Thus, 179 patients, 98 men and 81 women, aged $61 \pm 11$ years (mean $\pm \mathrm{SD}$ ), were enrolled in this study after giving informed consent. One hundred ten patients had undergone surgery for aortic valve replacement (AVR), 39 for mitral valve replacement (MVR), and the remaining 30 for both aortic and mitral valve replacement (DVR). The interval between transcranial Doppler monitoring and valve insertion was 15 months (13-17 months; median and $95 \%$ confidence intervals). All patients were receiving warfarin at the time of the transcranial Doppler study (target international normalized ratio 3-3.5). The actual international normalized ratio at the time of monitoring was not evaluated.

Methods. A detailed neurologic examination was performed in all cases. Subsequently, the middle cerebral arteries were identified at a depth of 52 to $58 \mathrm{~mm}$ and continuously insonated for 60 minutes with the 2-MHz transducers of a pulsed ultrasound machine (Multi-Dop X-4; DWL Elektronische Systeme GmbH, Sipplingen, Germany). Settings of the Doppler machine were as follows: power $110 \mathrm{~mW}$, sample volume 5 to $8 \mathrm{~mm}$, low-pass filter $80 \mathrm{kHz}$, high-pass filter 100 $\mathrm{Hz}$, and sweep 5 seconds. These settings were used throughout the study. Bilateral monitoring was not feasible in 17 patients because of insufficient temporal bone window on one side. All monitoring sessions were evaluated online and recorded on digital audiotapes using an 8-channel digital audiotape recorder (TASCAM DA 88; TASCAM America, Montebello, Calif) for later re-evaluation. This was performed by assigning 15 random tapes (30 hours of monitoring data) to a second examiner, blinded both to the clinical details of the involved patients and to the results of the first observer. Both observers were asked to note the exact position of each detected MES, based on the display of the digital audiotape recorder. MES were identified according to criteria established in a recent consensus. ${ }^{7}$ Their count was expressed as mean between the right and left middle cerebral arteries in bilaterally monitored patients. Otherwise, the MES count detected in the monitored middle cerebral artery was used.

Statistical analysis. Normally distributed data were expressed as mean \pm standard deviation $(\mathrm{SD})$ and compared by means of the 2-sample $t$ test. Non-normally distributed data were expressed as median and $95 \%$ confidence intervals and compared by means of the Mann-Whitney $U$ test. The hypothesis that MES counts significantly differed depending on valve position was tested with the Kruskal-Wallis test for each valve type. The $\chi^{2}$ test was used for comparison of frequency distributions; when the sample size was low, the Fisher exact test was used instead. The Spearman rank correlation was used to examine the influence of valve duration and valve size on MES counts.
A binary logistic regression model (using Minitab version 13) was applied to evaluate the influence of valve type, valve position, duration of valve implantation, cardiac rhythm, prevalence of neurologic complications, and presence of diabetes mellitus and arterial hypertension on the presence or absence of MES. All predictors were included as categorical factors, except for duration of valve implantation (continuous variable). For this model, MES counts were expressed as 0 (for MES-negative patients) or 1 (for MES-positive patients).

Interobserver variability was evaluated by means of the Cohen $\kappa$ statistic. ${ }^{8}$ Values derived from this evaluation range between -1 (complete disagreement) and 1 (complete agreement). A value of 0 reflects lack of a relation between the evaluations of the 2 observers. Acceptable to good agreement is indicated by values between 0.4 and 0.75 , and values greater than 0.75 indicate an excellent agreement. ${ }^{8}$

\section{Results}

No significant differences in age or sex were evident between patients having SJM and those having ATS valves. The duration of valve implantation was longer in patients with SJM as compared with ATS valves. This difference was not statistically significant when patients were split into subgroups according to the different valve positions, but it reached statistical significance when all patients were compared. The size of the implanted valves did not significantly differ between the 2 groups (Table I). Additionally, prevalence of arterial hypertension and diabetes mellitus was similar for both groups $(21 \%$ and $19 \%$ for patients with ATS and $22 \%$ and $15 \%$ for patients with SJM valves, respectively; $P=.91$ and $P=.48, \chi^{2}$ test). Heart rhythm was sinus in $60.4 \%$ and $55.7 \%$ and atrial fibrillation in $34.1 \%$ and $36.4 \%$ of patients with ATS and SJM valves, respectively; the remaining patients $(5.5 \%$ and $7.9 \%$, respectively) had a pacemaker. These differences were also insignificant $(P=.72)$.

MES counts were higher in patients with SJM valves than in patients with ATS valves. This difference was statistically significant when all patients were included in the analysis and also in the subgroups of patients with AVR and DVR (Table I). Statistically significant differences in MES prevalence in association with the valve type were observed in the total patient population $\left(\chi^{2}\right.$ test, $P=.009$; AVR vs MVR, $P=.1$; AVR vs DVR, $P=.004$; MVR vs DVR, $P=.2$ ). In the group of patients with ATS valves, no significant differences in MES prevalence were evident depending on the valve position $\left(\chi^{2}\right.$ test among all patients, $P=.1 ; \chi^{2}$ test, AVR vs MVR, $P=.1$; AVR vs DVR, $P=.09$; MVR vs DVR, $P=.8)$. The contrary was true in patients with SJM valves $\left(\chi^{2}\right.$ test among all patients, $P=.03 ; \chi^{2}$ test, AVR vs MVR, $P=.4$; Fisher exact test, AVR vs DVR, 
Table I. Clinical details and MES counts and prevalence in 179 patients with prosthetic heart valves

\begin{tabular}{|c|c|c|c|c|c|c|c|c|}
\hline Position & $N$ & $A g e^{*}(y)$ & $\operatorname{Sex}(F / M)$ & Duration $\dagger(\mathrm{mo})$ & Size* & MES per hourt & $\operatorname{MES}(\%)$ & Neuro (\%) \\
\hline \multicolumn{9}{|l|}{ AVR } \\
\hline ATS & 55 & $62 \pm 11$ & $22 / 33$ & $12(11-15)$ & $23 \pm 3$ & $0.8(0.3-1.5)$ & 42 & 7.3 \\
\hline SJM & 55 & $61 \pm 10$ & $16 / 39$ & $15(12-18)$ & $24 \pm 2$ & $8.5(3.8-13.8)$ & 67 & 3.6 \\
\hline$P$ value & & $.7 \ddagger$ & $.2 \S$ & $.1 \|$ & $.46 \ddagger$ & .0009 & $.007 \S$ & $.7 \Phi$ \\
\hline \multicolumn{9}{|l|}{ MVR } \\
\hline ATS & 21 & $61 \pm 11$ & $15 / 6$ & $18(12-19)$ & $28 \pm 2$ & $3.5(1-19.7)$ & 62 & 19 \\
\hline SJM & 18 & $58 \pm 12$ & $8 / 10$ & $22(14-29)$ & $28 \pm 3$ & $10(3.5-23.5)$ & 78 & 11.1 \\
\hline$P$ value & & $.4 \$$ & $.09 \S$ & $.7 \|$ & $.64 \ddagger$ & .3 & $.3 \S$ & $.7 \mathbb{I}$ \\
\hline \multicolumn{9}{|l|}{ DVR } \\
\hline ATS & 15 & $64 \pm 10$ & $11 / 4$ & $12(8-25)$ & $22 \pm 2 / 28 \pm 2$ & $2.9(1-9.8)$ & 67 & 6.7 \\
\hline SJM & 15 & $58 \pm 12$ & $9 / 6$ & $21(15-29)$ & $22 \pm 2 / 27 \pm 2$ & $30(10-57.5)$ & 100 & 13.4 \\
\hline$P$ value & & $.2 \ddagger$ & $.4 \S$ & .09 & $.76 / .57 \ddagger$ & .004 & .04 & 19 \\
\hline \multicolumn{9}{|l|}{ All } \\
\hline ATS & 91 & $62 \pm 10$ & $48 / 43$ & $13(12-16)$ & & $1.5(1-2.5)$ & 51 & 9.9 \\
\hline SJM & 88 & $60 \pm 11$ & $33 / 55$ & $18(15-20)$ & & $11(8-16.3)$ & 75 & 6.8 \\
\hline$P$ value & & $.21 \%$ & $.2 \S$ & .03 & & $.00001 \|$ & $.0007 \S$ & $.5 \S$ \\
\hline
\end{tabular}

$A V R$, Aortic valve replacement; $M V R$, mitral valve replacement; $D V R$, dual (aortic and mitral) valve replacement.

$*$ Data expressed as mean \pm standard deviation.

$\dagger$ Data expressed as median (95\% confidence intervals).

¥Two-sample $t$ test.

$\S \chi^{2}$ Test.

Mann-Whitney $U$ test.

IFisher exact test.

$P=.007$; Fisher exact test, MVR vs DVR, $P=.1$ ). The Kruskal-Wallis test revealed no significant differences in MES counts depending on valve position in patients with ATS or patients with SJM valves $(P=.6$ and $P=.3$, respectively). The exact MES counts for each position are listed in Table I.

The Spearman rank correlation revealed a significant relationship between duration of valve implantation and MES counts when data from all patients were analyzed $(\rho=0.25, P=.001)$. This was also true for patients with SJM valves $(\rho=0.3, P=.005)$, but not for patients with ATS valves $(\rho=0.09, P=.41)$.

MES counts did not correlate with valve size, except in patients with an SJM valve in the aortic position (AVR overall, $\rho=0.19, P=.05$; ATS valves, $\rho=-0.001, P=.994$; SJM valves, $\rho=0.29, P=.03$; MVR overall, $\rho=0.07, P=.66$; ATS valves, $\rho=0.086$, $P=.71$; SJM valves, $\rho=0.09, P=.73$; Spearman rank correlation).

Results of logistic regression analysis are displayed in Table II. Valve type was a significant predictor of MES counts, with SJM valves being a predictor of higher MES counts than ATS valves; presence of diabetes mellitus was a further significant predictor for lower MES counts.

Linear regression analysis demonstrated a strong correlation between MES counts in the right as compared with the left middle cerebral artery $\left(r^{2}=0.8, P<\right.$
.0001). The highest observed discrepancy was $800 \%$ (1 MES in the right and 8 in the left middle cerebral artery). The Cohen $\kappa$ statistic revealed an excellent agreement between the 2 observers $(\kappa=0.82)$.

A total of 15 patients had had neurologic symptoms since valve insertion. These consisted of amaurosis fugax $(n=3)$, transient ischemic attack $(n=4)$, and ischemic stroke $(n=8)$. All transient ischemic attacks involved the anterior circulation. Ischemic strokes involved the anterior circulation in 6 patients (partial anterior circulation syndrome in all cases) and the posterior circulation in 2 patients (isolated brainstem infarction, $\mathrm{n}=1$; brainstem and cerebellar infarction, $\mathrm{n}=1$ ). Six of the 15 patients had SJM valves and 9 had ATS valves $\left(\chi^{2}\right.$ test, $\left.P=.5\right)$. The aortic valve was replaced in 6 patients $(6 / 110,5.4 \%)$, the mitral in a further 6 patients $(6 / 39,15.3 \%)$, and both valves in 4 patients $(4 / 26,15.3 \%)$; these differences did not reach statistical significance (all patients, $\chi^{2}$ test, $P=.1$, AVR vs DVR, $P=.22$; AVR vs MVR, $P=.08$; MVR vs DVR, $P=1$; all Fisher exact test).

History of cerebral embolic complications after valve insertion was not a predictor of MES counts (Table II). Additionally, no statistically significant differences in MES counts were evident on direct comparison between patients with a history of cerebral embolic complications and the remaining patients $(17$ [8-31] vs 10 [5-18], $P=.1$; Mann-Whitney $U$ test). 
Table II. Evaluation of independent predictors of MES counts using binary logistic regression analysis in 179 patients with prosthetic heart valves

\begin{tabular}{lcccc}
\hline Variables & Coefficient & $S E$ & $95 \%$ CI & P value \\
\hline Constant & 0.08 & 0.59 & - & $1.78-7.66$ \\
Valve type (ATS/SJM) & 1.31 & 0.37 & $0.21-1.34$ & .001 \\
Valve position (AVR/MVR) & -0.62 & 0.47 & $0.73-10.78$ & .18 \\
Valve position (MVR/DVR) & 1.03 & 0.69 & $0.98-1.05$ & .14 \\
Valve duration (mo) & 0.01 & 0.02 & $0.7-14.9$ & .47 \\
Cerebral embolic events (yes/no) & 1.17 & 0.78 & $0.11-0.67$ & .13 \\
Diabetes mellitus (yes/no) & -1.32 & 0.43 & $0.32-1.72$ & .005 \\
Arterial hypertension (yes/no) & -0.3 & 0.43 & $0.77-3.93$ & .49 \\
Heart rate (SR vs AF) & 0.55 & 0.69 & $0.11-1.62$ & .21 \\
Heart rate (AF vs paced) & -0.87 & & & \\
\hline
\end{tabular}

$A V R$, Aortic valve replacement; $M V R$, mitral valve replacement; $D V R$, dual (aortic and mitral) valve replacement; $S R$, sinus rhythm; $A F$, atrial fibrillation; $S E$, standard error; $C I$, confidence intervals.

\section{Discussion}

The main findings of this study were the significant differences in MES counts between patients with SJM and those with ATS valves. This finding is further supported by the results of logistic regression analysis, demonstrating valve type as an independent predictor of MES counts. Only 1 previous study compared MES counts in patients with SJM $(\mathrm{n}=200)$ and ATS valves $(n=61)$. No significant differences in MES counts were noted between the 2 groups, although a tendency toward higher MES counts in patients with SJM valves was evident. ${ }^{5}$ The MES counts reported for SJM valves were lower than the ones observed in the present study (4 [3-6] and 11 [8-16], respectively). This was probably due to the fact that all patients with SJM valves were examined within 6 months after valve insertion in the previous study, as compared with a median duration of 18 months in our study. It thus appears probable that the discrepancies in our results and the ones previously reported are due to methodologic issues.

The differences in MES counts between patients with ATS and patients with SJM valves are difficult to explain assuming formed underlying embolic material, particularly because the 2 patient groups were comparable in their clinical characteristics, and the same anticoagulant regimen was used for all patients. Recent reports from several research groups add weight to the hypothesis that the underlying material of MES in patients with prosthetic valves consists of nitrogen bubbles. The observed significant differences in MES counts between 2 valve types that are quite similar in design and material composition suggest that even slight differences in the local fluid mechanical development in the vicinity of the bileaflet valve can have a profound influence on the flow field distant to the valve. The hydrodynamic characteristics of SJM and ATS valves were compared in 2 previous studies: similar pressure drops across both valves were observed in the first study, under both steady and pulsatile flow. Nevertheless, pressure drop was lower in the ATS valve as compared with the SJM valve under incorporation of an enlargement instead of a straight downstream conduit. ${ }^{9}$ Significant differences in mean increasing pressure drops and effective orifice areas were noted in the second study (both ATS > SJM) ${ }^{10}$ Still, both reports examined only hydrodynamic performance with the valve in the mitral position. The causative role of the aforementioned hydrodynamic findings in the discrepancies in MES counts observed in this study remains to be evaluated.

The incidence of embolic complications in patients with SJM valves was examined in several studies ${ }^{11-13}$ and reported between $1.09 \%{ }^{11}$ and $2.45 \%{ }^{13}$ per patientyear. Two clinical reports concerning the ATS valve have been published to date. One thromboembolic complication was noted in the first, describing 200 patients followed up for 1 to 3 years. ${ }^{14}$ A single cerebral embolic complication is also described in the second report, in which results from 100 patients, followed up for 5 to 27 months, are presented. ${ }^{15}$ Obviously, the small size of the aforementioned reports prohibits clinical comparisons between the 2 groups. The prevalence of cerebral embolic complications noted in our study is markedly higher than previously reported. It must be taken into account, though, that most symptoms were transient. In fact, most patients with transient ischemic attacks and amaurosis fugax reported these incidents only after detailed questioning, having denied neurologic complications beforehand. Considering the small sample size of the present study, 
definitive statements on the prevalence of cerebral embolic events are not warranted. Additionally, our patients were asked to attend the ultrasound examination on an outpatient basis; this fact may have biased our results, because only healthy or at least not heavily disabled patients were bound to attend. We presume that the high prevalence of amaurosis fugax in our study is due to the fact that the ophthalmic artery, with its small diameter, missing collaterals, and dramatic neurologic signs, represents a predilection site for symptomatic embolism.

MES counts were not associated with a history of cerebrovascular events in our study, which corresponds to the results of most previous studies. Obviously, patients were not examined immediately after the cerebrovascular events occurred, but in some cases as long as 2 years later, so that a potential relationship in the acute phase cannot be excluded. On the other hand, MES counts in patients with prosthetic heart valves remain relatively constant over time. ${ }^{16}$

Duration of valve implantation was not identified as an independent predictor of MES counts in logistic regression analysis. Still, results from nonparametric correlation demonstrated a significant influence of valve duration on MES counts in patients with SJM valves but not in those with ATS valves. This finding is difficult to explain, because it suggests a significant difference in the behavior of the 2 valves. It must be emphasized that only 1 previous study examining a homogeneous group, namely patients with Carbomedics valves (Sulzer Carbomedics, Inc, Austin, Tex), described such an influence. ${ }^{2}$ It has been observed that the incidence of MES increases with time of implantation for porcine valves implanted in an animal model, due to increasing pannus overgrowth on the valve. ${ }^{17} \mathrm{It}$ is possible that a small difference in tissue encroachment between the ATS and SJM valves may produce a similar effect with time. Although it appears possible that the influence of valve duration on MES counts differs depending on the valve type, we are unable to provide definitive information on this matter.

No previous study has examined the influence of diabetes mellitus on MES counts. The underlying mechanism of this potential effect is unclear. It may be that blood osmolality influences formation, as well as halflife, of cavitation bubbles, thus affecting MES counts. Obviously, several additional parameters would have to be included in future analyses, particularly adequacy of diabetes control and actual blood glucose level at the time of transcranial Doppler study.

Influence of MES on neuropsychologic deficit in patients with prosthetic valves was suggested in a recent study. ${ }^{6}$ However, the sample size of this study was quite small, thus prohibiting definitive statements on this matter. Provided that large-scale studies confirm the influence of MES on cognitive function, this method is bound to become a standard test for these patients, at least in the evaluation of new valve types.

In conclusion, our study provided evidence of significant differences in MES counts between patients with ATS and SJM valves. A relation with the prevalence of neurologic complications could not be established. Influence of MES on cognitive function remains to be evaluated.

Received for publication May 26, 2000; revisions requested June 26, 2000; revisions received Aug 24, 2000; accepted for publication Nov 28, 2000.

Address for reprints: D. Georgiadis, MD, Department of Neurology, University of Heidelberg, Im Neuenheimer Feld 110, 691210 Heidelberg, Germany (E-mail: dimitrios.georgiadis@medizin.uni-halle.de).

\section{REFERENCES}

1. Sliwka U, Diehl RR, Meyer B, Schöndube F, Noth J. Transcranial Doppler "high intensity transient signals" in the acute phase and long-term follow-up of mechanical heart valve implantation. J Stroke Cerebrovasc Dis 1995;5:139-46.

2. Braekken KS, Russell D, Brucher R, Svennevig J. Incidence and frequency of cerebral embolic signals in patients with a similar bileaflet mechanical heart valve. Stroke 1995;26:1225-30.

3. Georgiadis D, Grosset DG, Kellman AW, Faichney A, Lees KR. Prevalence and characteristics of intracranial microemboli signals in patients with different types of prosthetic cardiac valves. Stroke 1994;25:587-92.

4. Georgiadis D, Kaps M, Berg J, Mackay TG, Herlein FW, Wheatley DJ, et al. Transcranial Doppler detection of microemboli in prosthetic heart valve patients: dependency upon valve type. Eur J Cardiothorac Surg 1996;10:253-8.

5. Sliwka U, Georgiadis D. Clinical correlations of Doppler microemboli signals in patients with prosthetic heart valves: analysis of 580 cases. Stroke 1998;29:140-3.

6. Deklunder G, Roussel M, Lecroart JL, Prat A, Gautier C. Microemboli in cerebral circulation and alteration of cognitive abilities in patients with mechanical prosthetic heart valves. Stroke 1998;29:1821-6.

7. Consensus Committee of the Ninth International Cerebral Hemodynamics Symposium. Basic identification criteria of Doppler microembolic signals. Stroke 1995;26:1123.

8. Fleiss JL. Statistical methods for rates and proportions. In: Statistical methods and proportions. 2nd ed. New York: John Wiley; 1981. p. 212-2.

9. Feng Z, Umezu M, Fujimoto T, Tsukahara T, Nurishi M, Kawaguchi D. In vitro hydrodynamic characteristics among three bileaflet valves in the mitral position. Artif Organs 2000;24:34654.

10. Verdonck PR, Van Nooten GJ, Van Belleghem Y. Pulse duplicator 
hydrodynamics of four different bileaflet valves in the mitral position. Cardiovasc Surg 1997;5:593-603.

11. Baudet EM, Puel V, McBride JT, Grimaud JP, Roques F, Clerc F, et al. Long-term results of valve replacement with the St. Jude Medical prosthesis. J Thorac Cardiovasc Surg 1995;109: 858-70.

12. Burckhardt D, Striebel D, Vogt S, Hoffmann A, Roth J, Weiss P, et al. Heart valve replacement with St. Jude Medical valve prosthesis: long-term experience in 743 patients in Switzerland. Circulation 1988;78(3 Pt 2):I-18-24.

13. Kinsley RH, Antunes MJ, Colsen PR. St. Jude Medical valve replacement: an evaluation of valve performance. J Thorac Cardiovasc Surg 1986;92(3 Pt 1):349-60.
14. Westaby S, Van Nooten G, Sharif H, Pillai R, Caes F. Valve replacement with the ATS open pivot bileaflet prosthesis. Eur J Cardiothorac Surg 1996;10:660-5.

15. Van Nooten G, Caes F, Francois K, Missault L, Van Belleghem Y. Clinical experience with the first 100 ATS heart valve implants. Cardiovasc Surg 1996;4:288-92.

16. Georgiadis D, Kaps M, Siebler M, König M, Hill M, Berg J, et al. Variability of Doppler microemboli signal counts in patients with prosthetic cardiac valves. Stroke 1995;26:439-43.

17. Raco L, Belcher PR, Sim I, McGarrity A, Bernacca GM, Wheatley DJ. Platelet aggregation and High-Intensity Transient Signals (HITS) in a sheep model of mitral valve replacement. J Heart Valve Dis 1999;8:476-80.

Access to The Journal of Thoracic and Cardiovascular Surgery Online is reserved for print subscribers!

Full-text access to The Journal of Thoracic and Cardiovascular Surgery Online is available for all print subscribers. To activate your individual online subscription, please visit The Journal of Thoracic and Cardiovascular Surgery Online, point your browser to http://www.mosby.com/jtcvs, follow the prompts to activate your online access, and follow the instructions. To activate your account, you will need your subscriber account number, which you can find on your mailing label (note: the number of digits in your subscriber account number varies from 6 to 10). See the example below in which the subscriber account number has been circled:

\section{Sample mailing label}

\begin{tabular}{l|l|}
\cline { 2 - 3 } $\begin{array}{l}\text { This is your subscription } \\
\text { account number }\end{array}$ & $\begin{array}{l}* * * * * * * * * * * * * * * * * * * * * * * * * * 3-D I G I T \\
\text { SJ P1 } \\
\text { SEB00 J027 C: 1 1234567-89 U 05/00 Q: } 1\end{array}$ \\
J. H. DOE, MD \\
531 MAIN ST \\
CENTER CITY, NY 10001-001
\end{tabular}

Personal subscriptions to The Journal of Thoracic and Cardiovascular Surgery Online are for individual use only and may not be transferred. Use of The Journal of Thoracic and Cardiovascular Surgery Online is subject to agreement to the terms and conditions as indicated online. 